Cinémas

Revue d'études cinématographiques

Revue d'études cinématographiques

Journal of Film Studies

\title{
Dis-moi comment tu filmes - et comment tu montes - tes dialogues et je te dirai quel genre de film tu réalises
}

\section{Jan Baetens}

Volume 13, numéro 1-2, automne 2002

Limite(s) du montage

URI : https://id.erudit.org/iderudit/007961ar

DOI : https://doi.org/10.7202/007961ar

Aller au sommaire du numéro

Éditeur(s)

Cinémas

ISSN

1181-6945 (imprimé)

1705-6500 (numérique)

Découvrir la revue

Citer cet article

Baetens, J. (2002). Dis-moi comment tu filmes - et comment tu montes - tes dialogues et je te dirai quel genre de film tu réalises. Cinémas / Revue d'études cinématographiques, 13(1-2), 165-183. https://doi.org/10.7202/007961ar
Résumé de l'article

Le présent article se propose d'analyser le jeu du two-shot et du champ-contrechamp dans une scène dialoguée tirée des quatre films suivants : It Happened One Night, Bringing Up Baby, Orlando et The Color Purple. Son propos est triple : montrer que la manière de filmer un dialogue peut ne pas se réduire à l'enchaînement traditionnel " two-shot + champ-contrechamp "; indiquer que l'interprétation traditionnelle de ces deux types de plans que proposent les théoriciens du montage invisible (two-shot = plan d'ensemble ou establishing shot, champ-contrechamp = action) est parfois insuffisante ; suggérer, essentiellement à des fins didactiques, que le dialogue filmé peut fonctionner, surtout dans la perspective d'une didactique de l'analyse du cinéma, comme une sorte de modèle réduit du film qui l'incorpore. 


\section{Dis-moi comment tu filmes et comment tu montes tes dialogues et je te dirai quel genre de film tu réalises}

\section{Jan Baetens}

\section{RÉSUMÉ}

Le présent article se propose d'analyser le jeu du two-shot et du champ-contrechamp dans une scène dialoguée tirée des quatre films suivants: It Happened One Night, Bringing Up Baby, Orlando et The Color Purple. Son propos est triple: montrer que la manière de filmer un dialogue peut ne pas se réduire à l'enchaînement traditionnel "two-shot + champ-contrechamp "; indiquer que l'interprétation traditionnelle de ces deux types de plans que proposent les théoriciens du montage invisible (two-shot = plan d'ensemble ou establishing shot, champcontrechamp = action) est parfois insuffisante; suggérer, essentiellement à des fins didactiques, que le dialogue filmé peut fonctionner, surtout dans la perspective d'une didactique de l'analyse du cinéma, comme une sorte de modèle réduit du film qui l'incorpore.

\section{ABSTRACT}

The present article proposes to analyze the roles of the establishing shot and of the shot reverse shot in a dialogued scene from the four following films: It Happened One Night, Bringing Up Baby, Orlando and The Color Purple. The intention is threefold: to show that, when filming dialogue, it is possible not to revert to the traditional formula "establishing shot + shot reverse shot;" to show why the traditional interpretation of these two types of shots as offered by theorists of invisi- 
ble editing (establishing shot $=$ long shot, shot reverse shot $=$ action) is sometimes insufficient; to suggest, essentially for didactic purposes, that a filmed dialogue can operate, especially from the didactic perspective of cinema analysis, as a sort of condensed model for the film in which it is contained.

\section{Le dialogue filmé, un sujet simple mais problématique}

La question du dialogue au cinéma, qui est un problème auquel tout metteur en scène se voit tôt ou tard confronté, reste un objet théorique relativement négligé. Certes, l'importance des dialogues est reconnue par tous ceux qui s'intéressent au scénario ${ }^{1}$. De même, il existe de brillantes analyses qui prennent les dialogues du film, pour banals qu'ils paraissent, tout à fait (et pertinemment) au sérieux ${ }^{2}$. Et la plupart des ouvrages de base sur le septième art consacrent au moins quelques notes plus ou moins fournies à la question (mais souvent dissimulées à l'intérieur d'un long développement sur le point de vue ${ }^{3}$ ). Toutefois, ce qui est commun à la plupart de ces études, c’est le relatif désintérêt pour la représentation visuelle du dialogue, c'est-à-dire pour la manière de filmer un dialogue 4 . En effet, ou bien l'analyste se contente de quelques généralités sur les rapports entre dialogue et point de vue, l'analyse du dialogue étant vite reléguée au second plan au profit de considérations plus vastes sur les techniques les plus fréquemment utilisées pour filmer une action; ou bien il se concentre sur le traitement très particulier du dialogue dans tel ou tel film ${ }^{5}$, sans construire une approche globale du phénomène. Bref, selon les cas, on généralise trop ou pas assez, si bien que l'analyse du dialogue filmé reste un des parents les plus pauvres qui soient de l'analyse du langage cinématographique.

Le peu d'attention au dialogue filmé résulte à mon sens de la tension entre "mots" et "images" qui continue à hanter la critique et la théorie du cinéma. Tout se passe un peu comme si l'analyse du dialogue ravivait à son corps défendant le choc créé par l'avènement du parlant et, partant, la crainte, du moins chez certains, de voir le cinéma de nouveau réduit à n'être que du théâtre filmé. Comme si le fait même de se pencher sur les manières de filmer un dialogue était une façon subreptice ou involontaire d'admettre que le cinéma reste plus tributaire du modèle 
thêâtral qu'on ne le voudrait parfois. Cette crainte d'un retour de modèles hérités du thêâtre n'était certes pas seulement liée à l'avènement du parlant en lui-même (après tout, le cinéma muet avait parfaitement intégré un grand nombre d'accompagnements sonores), mais aussi à la fixité de la caméra imposée par les nécessités de la prise du son en direct (ces contraintes ne se dissiperont que dans la décennie suivante, avec l'introduction d'un matériel plus léger). Il n'empêche cependant que, par suite d'une confusion dont les polémiques sont rarement exemptes, la "décadence» du cinéma dénoncée dans les années 1930 est souvent mise sur le compte du seul parlant, qui sert en quelque sorte de bouc émissaire et qui attire sur lui les foudres de tous les «déçus du cinéma».

Historiquement parlant, toutefois, le rapport entre dialogue filmé et dialogue théâtral est incontestable. Comme cinéma d'attractions, le cinéma des premiers temps était d'abord un spectacle visuel qui devait trouver sa place dans un ensemble de représentations foraines et thêâtrales, qui lui servaient à la fois de modèle et de repoussoir. La dimension proprement théâtrale de ce cinéma reste éclatante, même en faisant abstraction du recours aux intertitres ou des conventions régissant l'entendement des mots (inaudibles mais parfaitement compréhensibles ${ }^{6}$ ) prononcés par les acteurs à l'écran. Le cas très particulier du "film d'art", avec sa tentative de transposer les codes du théâtre bourgeois à l'univers moins prestigieux du cinéma, ne doit pas dissimuler qu'en fait tout le cinéma muet était une pratique culturelle traversée d'un bout à l'autre par les stéréotypes et l'imaginaire du spectacle vivant. La discussion née de l'introduction du parlant (et dont on sait qu'elle renaît de temps à autre, chaque fois que l'essence ou la pureté du média est jugée en danger) se trompe donc fondamentalement de cible: la dimension parlée ou théâtrale ne peut être ce qui menace le cinéma parlant, puisque même à l'époque du muet (et, ici aussi, abstraction faite de ce que nous avons appris ces trente dernières années sur les sons du muet), le cinéma était déjà largement soumis à des codes non cinématographiques, en l'occurrence verbaux et théâtraux. Bref, le cinéma ne doit pas avoir peur de devenir intermédiatique, pour la bonne et simple raison qu'il l'a toujours été (c'est même plutôt des "puristes » qu'il conviendrait 
de le sauver, mais ceci est un autre débat), et les récentes recherches sur l'histoire des médias comme "remédiation ${ }^{7}$ " ou en termes de "double naissance ${ }^{8}$ " montrent à l'envi que le dialogue filmique n'est pas quelque chose qui a été réintroduit dans le cinéma, mais au contraire quelque chose dont le cinéma est parti pour en faire (ou non) quelque chose d'autre.

\section{Le dialogue filmé : un modèle unique?}

Ce qui change lorsqu'on passe de la représentation du dialogue théâtral à celle du dialogue au cinéma, les outils traditionnels de l'analyse filmique permettent de l'articuler clairement. Sur le plan profilmique de la mise en scène, l'unité matérielle de lieu cesse d'être une (presque-)fatalité ou, plus exactement peut-être, une (quasi-)évidence : les sujets dialoguant peuvent être montrés en divers endroits, par exemple lorsqu'ils sont en train de se téléphoner, ou sont capables de se déplacer sans coupure d'un endroit donné à un endroit tout autre, par exemple lorsqu'ils se trouvent dans une voiture. Sur le plan filmique de la mise en film, le point de vue n'est plus celui, unique et en principe immobile, d'un spectateur dans la salle, mais celui, certes "incomplet" en raison des "restrictions de champ ", que donne une caméra devenue rapidement mobile, à la fois en mesure d'occuper plusieurs points dans l'espace et capable d'adopter plusieurs angles et distances par rapport à l'objet filmé. Sur le plan postfilmique du montage enfin, l'unité formelle de la scène dialoguée peut être remplacée par des structures plus complexes. Bref, de nombreuses modifications de la situation initiale, à savoir le dialogue théâtral, deviennent possibles, sans pour autant s'imposer comme absolument indispensables, de sorte que filmer un dialogue revient toujours à osciller entre deux extrêmes: d'une part, une représentation "statique", qui renonce le plus possible aux manipulations de mise en scène, de mise en film et de mise en chaîne; d'autre part, une représentation qui multiplie les interventions filmiques, profilmiques et postfilmiques.

Très tôt, pourtant, une certaine norme semble s'installer tout en se dérobant non moins rapidement à l'attention du spectateur. C'est l'équivalent du montage invisible dans le cinéma 
narratif traditionnel de Hollywood (la caméra suit les personnages, adopte leur point de vue et fait tout pour aspirer le spectateur dans l'histoire au lieu d'attirer le regard sur ses propres artifices). Pour le dialogue, ce principe du montage invisible prend les formes suivantes: sur le plan de la mise en scène, les personnages ne jouent pas pour une salle, représentée virtuellement par la caméra, mais se parlent les uns aux autres, traitant la caméra comme un objet tabou. Sur le plan de la mise en film, les dialogues sont représentés en plan subjectif ou, plus exactement, en plan semi-subjectif (dont les plans "par-dessus l'épaule» sont un bon exemple), pour ce qui concerne tant le point de vue que la distance et l'angle de vue. Sur le plan de la mise en chaîne, le dialogue se coule imperceptiblement dans le moule du montage alterné, en l'occurrence selon la technique du champ-contrechamp, qui va concentrer sur lui un ensemble de propriétés impressionnantes : le champ-contrechamp finit par être utilisé comme un terme désignant tout à la fois un type de cadrage, un type de point de vue et un type de raccord, à tout le moins en s'agrégeant un ensemble d'autres types de plans et d'images qui mériteraient sans doute une approche moins homogénéisante (les vues "par-dessus l'épaule», par exemple, sont parfois intégrées à la catégorie englobante du champcontrechamp, cependant que tous les types d'images qui ne sont pas susceptibles d'être annexés par le label englobant du champcontrechamp sont pris en charge par la notion non moins écrasante de plan d'ensemble ou establishing shot ${ }^{9}$ ). Combinées presque automatiquement, ces données filmiques, profilmiques et postfilmiques façonnent une manière de filmer les dialogues qui se laisse facilement naturaliser : le personnage A qui parle est vu à travers le regard du personnage $\mathrm{B}$ auquel il s'adresse, et vice versa. Ainsi se trouve confirmée de nouveau l'intuition fondamentale de Laura Mulvey, qui avait basé sa critique féministe du cinéma hollywoodien sur l'écrasement des différences entre les regards de la caméra, du personnage et du spectateur ${ }^{10}$ : d'une part la caméra adopte le point de vue du personnage, d'autre part le spectateur est conduit à s'identifier à ce même personnage, de sorte que toute distance critique entre les trois instances du regard au cinéma se trouve comme évacuée au seul 
profit de ce qui se passe au niveau de la fiction narrative. La focalisation presque exclusive sur le champ-contrechamp comme technique de représentation des dialogues dans le type de cinéma visé par Mulvey est donc parfaitement compréhensible. Aucune autre technique ne semble aussi efficace dans la soumission de la caméra à la perspective du personnage et à l'identification du spectateur à son point de vue. Pour obtenir dans un dialogue filmé l'immersion dans l'univers fictif, l'identification au personnage diégétique et la sexualisation du regard, le champ-contrechamp paraît offrir toutes les garanties nécessaires, et il ne faut donc pas s'étonner que les discours sur le cinéma qui mettent en avant le corpus hollywoodien se plaisent à l'ériger en norme incontestable. (Inversement, il est non moins logique d'observer que le cinéma documentaire ou expérimental, dont il ne sera pas question dans cet article, recourt massivement à d'autres manières de filmer le dialogue.)

La raison principale du succès de la formule traditionnelle excède pourtant ce rapport avec le montage invisible et les implications idéologiques que l'on sait être maintenant les siennes: au-delà s'esquisse toujours la crainte de la rethéâtralisation rampante du cinéma, dont témoigne a contrario la technique du two-shot, c'est-à-dire de la représentation qui cadre non pas un seul des personnages en dialogue, mais bien les deux. Le système est tellement simple et le code devenu tellement familier, que toute autre façon de filmer les dialogues est soit ignorée (il n'y a aucun rapport entre le nombre finalement très élevé de two-shots dans le cinéma hollywoodien et le quasi-silence qui entoure cette technique dans la littérature spécialisée), soit perçue comme un écart par rapport à cette norme implicite (généralement, l'astuce naturalisante consiste à poser que le two-shot précède souvent le champ-contrechamp et que, comme tel, il est comparable au plan de présentation ou establishing shot qui précède pour la bonne compréhension des choses l'entrée en la matière narrative).

Ces stéréotypes ne sont pas faux. Le problème est seulement qu'ils dissimulent souvent la complexité de nombreuses œuvres, même hollywoodiennes. Les quelques exemples de scène en twoshot dont on aimerait discuter dans ces pages devraient montrer 
tout de suite que cette technique est utilisée de manière plus subtile et surtout plus articulée qu'on aurait peut-être tendance à le penser. Loin de se réduire à quelque syntagme figé: "twoshot + champ-contrechamp ", dont chaque élément possède sa propre signification canonique (two-shot $=$ plan d'introduction ou vue d'ensemble; champ-contrechamp = action vécue à travers les dialogues), les différentes manières de filmer un dialogue doivent se penser de façon nettement plus ouverte, tant sur le plan syntagmatique que sur le plan paradigmatique: en pratique, l'enchaînement de la séquence ne va pour ainsi dire jamais du two-shot au champ-contrechamp; il convient en revanche de voir à quel point chaque unité syntagmatique garde sinon la liberté, du moins la possibilité d'utiliser tous les éléments du paradigme du dialogue filmé (dont le champ-contrechamp et le two-shot ne sont que deux exemples parmi d'autres). Analyser comment un dialogue est filmé revient donc à se faire sensible à la manière dont syntagme et paradigme s'articulent, et quelle "idée" de la scène, voire du film, en émerge. Dis-moi comment tu filmes tes dialogues et je te dirai quel genre de films tu réalises...

Plus généralement, les exemples analysés cherchent aussi à suggérer que l'analyse du dialogue filmé est un des moyens les plus rapides et fiables de se faire une idée de la structure d'un film tout entier: ces passages-là, examinés à l'aune de critères qui passent trop souvent inaperçus, sont une véritable voie royale de l'analyse du cinéma. Du point de vue didactique, leur valeur heuristique est très forte. Dans les exercices de segmentation, qui restent le b.a.-ba de toute analyse filmique, cette approche peut contribuer puissamment à dépasser la seule division mécanique des séquences, tellement les dialogues aident à voir comment une scène est structurée à travers ses plans successifs.

Pour mener cette brève analyse, on s'appuiera ici sur deux films des années 1930, qui posent l'un et l'autre directement la persistance de modèles scéniques au moment où les talkies sont censés montrer qu'ils sont capables de faire plus et mieux que du théâtre filmé, et deux films plus récents, qui s'efforcent tous les deux d'interroger certains stéréotypes liés à la division sociale du regard, avec son clivage du sujet masculin et de l'objet féminin. 
Ces films ne constituent évidemment qu'un petit échantillon des œuvres qu'il conviendrait d'analyser pour vérifier de manière plus fine les idées défendues dans cet article. Leur choix, pourtant, n'a pas été fait au hasard, et on ose penser que les limites quantitatives du corpus ne nuisent pas trop à sa représentativité qualitative, en tout cas dans le domaine du cinéma narratif "commercial».

\section{Comme au théâtre?}

Miami-New York (It Happened One Night, Frank Capra, 1934), un des premiers exemples de la nouvelle comédie américaine des années 1930, n’offre à première vue qu'une application habile des thèmes et techniques hollywoodiens de l'époque. Le film raconte l'histoire d'amour entre une riche héritière (Ellie Andrews/Claudette Colbert), qui s'enfuit pour épouser un aviateur contre l'avis de son père, et un pauvre journaliste (Peter Warne/Clark Gable), qui l'aide dans sa fuite en se faisant passer pour son mari (d'abord pour lui être utile, ensuite parce qu'il flaire un scoop, enfin parce que...). Miami-New York, est pourtant aussi un film qui joue avec bien des conventions. En tant qu'il relève de la "screwball comedy", que l'on sait être, grâce entre autres à Stanley Cavell ${ }^{11}$, le genre qui a introduit davantage d'égalité dans les rapports entre hommes et femmes, le film dépasse déjà certains clichés sexistes. Et, comme réponse personnelle du réalisateur à l'autocensure imposée par le Hays Production Code, il est resté célèbre pour une scène de double strip-tease, d'abord masculin, puis féminin. Vers le début du film, les deux personnages, qui se connaissent encore à peine, sont obligés de partager la même chambre de motel. Pour créer un minimum de privacy, Gable tend une couverture («les murs de Jéricho") entre les deux lits, mais comme Colbert boude toujours et refuse de choisir un des deux lits, il commence à se déshabiller, provoquant d'abord la surprise du spectateur (il s'avère ne pas porter de chemise de corps, ce qui était du jamais vu dans le cinéma mainstream d'après le Code), puis, au moment où il s'apprête à enlever son pantalon, la fuite de Colbert. Celle-ci se déshabille alors derrière la couverture, ce qui est évidemment une manière de tout suggérer sans rien montrer. 
Cette scène clé de quelque six minutes fait un usage très subtil des différentes possibilités de filmer un dialogue (n'oublions pas que, comme beaucoup d'autres "screwball comedies", le scénario de ce film a de lointaines origines théâtrales). Si elle est tellement intéressante pour notre propos, ce n'est pas uniquement parce que la part du champ-contrechamp y est réduite, mais aussi et surtout parce qu'elle montre très bien à quel point la représentation d'un dialogue engage la totalité des éléments filmiques.

La représentation de ce passage est éminemment théâtrale. La chambre de motel est représentée comme une scène de théâtre, la caméra occupant grosso modo le "quatrième mur", la paroi invisible de la scène traditionnelle: en l'occurrence, cette scène a une porte d'entrée à droite (du point de vue du spectateur dans la salle), deux lits et deux fenêtres au fond, et un mur aveugle à gauche; aucune prise de vues n'est effectuée qui montre le côté de la chambre opposé au mur du fond (la seule exception est un court plan rapproché sur Ellie à la fenêtre, mais ce plan est filmé de l'extérieur et ne permet pas de voir le fond de la chambre). Ce dispositif éminemment théâtral est encore souligné par le maintien, presque tout au long du passage, du two-shot, d'une part, et d'un traitement très singulier du champ-contrechamp, d'autre part. Petit à petit, on passe certes de la représentation simultanée des deux personnages dans le même plan à leur représentation alternée, mais on se rend vite compte que cette transition n'a rien à voir avec le système conventionnel du plan d'ensemble suivi rapidement du champ-contrechamp (puis d'un nouveau plan d'ensemble ou reestablishing shot, etc.).

Au début, le two-shot, qui souligne très bien le caractère claustrophobique de la scène et la gêne d'Ellie de se retrouver dans le même espace (une chambre à coucher!) que Peter, s'ingénie à creuser les différences entre les deux personnages. Leur coprésence dans le même plan permet de mieux marquer ce qui les sépare que n'importe quel champ-contrechamp. Contrairement à Ellie, qui la plupart du temps est filmée en trois quarts ou frontalement et qui reste plutôt immobile dans la première partie de cette séquence, Peter change souvent de position devant la caméra (il est ainsi régulièrement montré de 
dos), à tel point qu'on a l'impression qu'il est en train de danser autour de la femme. Les différences ne s'arrêtent pas là: Peter dépasse Ellie de près d'une tête, et ses déplacements font que dans nombre de plans, il se trouve plus près de la caméra que celle-ci, qui devient ainsi encore plus menue; de plus, les vêtements de Peter sont plus clairs que les habits foncés d'Ellie, dont l'immobilité parait pour cette raison encore accrue. Ces différences entre les deux objets préparent la différenciation du regard du sujet (qu'il s'agisse du personnage diégétique ou, par ricochet, du spectateur, masculin ou féminin, dans la salle): peu à peu, la femme, ici particulièrement passive, s'institue en objet, tandis que l'homme, actif comme il se doit selon le code traditionnel du cinéma narratif hollywoodien, impose son rôle de sujet. Le champ-contrechamp n'apparaît que dans deux passages violemment érotisés: une première fois quand Peter invite Ellie à prendre le lit (à gauche de l'écran) qu'il lui a préparé, une seconde fois quand il installe la couverture entre les lits (au milieu donc de l'écran). Ce champ-contrechamp n'est pourtant pas très conventionnel, puisqu'il ne s'accompagne d'aucun raccord dans l'axe: si les plans où Peter apparaît seul sont «imaginables» à travers le regard d'Ellie, les plans où Ellie se trouve seule ne représentent nullement le point de vue de Peter, qui lui tourne le dos et qui semble se trouver à une distance plus grande que celle suggérée par l'image d'Ellie. Manifestement, la logique du champ-contrechamp se voit d'emblée déséquilibrée: autant les images montrant Peter peuvent être "naturalisées» comme des plans subjectifs représentant le regard d'Ellie, autant celles montrant Ellie résistent à cette facile naturalisation et pointent dans une tout autre direction (celle, déjà, de la caméra?).

Quand Peter s'apprête à enlever son pantalon, il oblige Ellie à s'enfuir de l'autre côté du "rideau » improvisé où elle commence à se déshabiller à son tour, mais non sans avoir demandé que Peter éteigne la lumière. Comme toutefois les fenêtres derrière les lits restent en partie ou totalement ouvertes, la scène n'est plongée que dans une sorte de pénombre: on verra que, de cette convention cinématographique aussi, Capra saura tirer des effets très concertés. Chacun des personnages une fois de son côté des "murs de Jéricho", puis au lit, le two-shot est relayé par des vues 
rapprochées des deux personnages, qui reprennent le dialogue après un certain temps. On observe d'abord que les rapports formels établis dans la première partie de la séquence s'inversent symétriquement: la femme apparaît souvent encore plus foncée que l'homme, elle est filmée de beaucoup plus près que lui et, surtout, la tension entre mobilité et immobilité se voit agencée tout autrement (autant Peter reste immobile au lit, autant Ellie semble maintenant très agitée). La transformation du point de vue confirme-t-elle ces changements? Le passage du two-shot (qui reviendra à la toute fin de la séquence, sous la forme d'un establishing shot terminal bien dans la logique de la narration hollywoodienne, qui marque ainsi la clôture paisible de la diégèse) à une série de champs-contrechamps, prolonge-t-il la manipulation du regard déjà entamée dans la première partie de la scène, où l'on a signalé l'émergence progressive d'un objet regardé et d'un sujet regardant?

De nouveau, les apparences sont trompeuses. L'alternance des plans sur les personnages isolés (Peter au lit, Ellie en train de se déshabiller, puis au lit) semble corroborer l'installation du regard subjectif d'un personnage sur l'autre, dans la plus parfaite des logiques narratives hollywoodiennes. En réalité, les divers champs-contrechamps creusent plutôt l'emploi curieux qui en était fait dans la première partie de la scène, avec leur dissymétrie frappante entre les vues de Peter (focalisées par Ellie, suppose-t-on) et celles d'Ellie (dues plus manifestement à la seule caméra, comme si quelque voyeur s'était introduit dans la chambre du motel). Ce qui se passe ici, c'est que les plans alternés qui montrent tantôt Peter et tantôt Ellie rompent absolument avec la convention du raccord dans l'axe des regards. Nous voyons bel et bien les deux personnages alternativement, mais ces plans ne sont jamais subjectifs, pour des raisons tant diégétiques (entre les lits a été tendue cette fameuse couverture, qui empêche l'homme et la femme de se voir réellement) que filmiques (Peter et Ellie ne sont pas filmés chacun leur tour du point de vue de l'autre, mais d'un point de vue totalement différent, celui de la caméra située au niveau du quatrième mur invisible, c'est-à-dire du point de vue qui pourrait être celui du spectateur d'une salle de théâtre, même si, en raison des 
changements de distance d'un plan à l'autre, ce spectateur hypothétique n'est pas immobile comme il le serait au théâtre). Le champ-contrechamp ne peut donc être considéré comme un moyen de naturaliser le montage, c'est-à-dire de le rendre invisible au spectateur (par exemple en l'invitant à partager le point de vue des personnages de la fiction ou, davantage encore, en inféodant son rythme et sa structure à la logique de la succession narrative et de la manière dont circule la parole d'un personnage à l'autre). Les images de Peter et Ellie ne peuvent être celles que les personnages voient eux-mêmes, elles sont doublement des images fictives. Elles représentent la manière dont les personnages s'imaginent l'un l'autre, tout en affichant aussi la présence de la caméra dont l'emplacement n'est pas récupérable en termes diégétiques. Une distance critique à l'égard de la psychologisation du regard est ainsi préservée, qui conduit à une rethéâtralisation très nette de la séquence: la dernière image de la scène dénote presque littéralement un dispositif théâtral, dont Capra montre à profusion qu'il n'est pas moins cinématographique que n’importe quel procédé soi-disant "spécifique», tel le champ-contrechamp.

Une courte comparaison avec une autre comédie du même genre, L'Impossible Monsieur Bébé (Bringing Up Baby, Howard Hawks, 1938) montre un emploi comparable du champcontrechamp et du two-shot ${ }^{12}$. Le premier tend en effet à se limiter plus qu'on ne le pense, sans aucun doute pour contrer les dérives inévitablement (?) sexistes de la différenciation du sujet regardant, par définition hollywoodienne masculin, et de l'objet regardé, supposé quant à lui féminin. Dans ce film, où les rapports de force traditionnels au sein du couple sont particulièrement mis à mal, un emploi trop systématique du champcontrechamp tendrait à détourner l'attention du public de la lutte entre homme et femme au profit d'une structure scopique plus conventionnelle qui accentuerait inéluctablement le sex-appeal du personnage féminin joué par Katherine Hepburn (le mâle de service étant un Cary Grant exceptionnellement falot et maladroit). Hawks opte pour une certaine distance par rapport au couple, que le two-shot semble plus à même de garantir que le champ-contrechamp. Pourtant, le two-shot est parfaitement intégré par Hawks à la diégèse comme au langage cinématogra- 
phique. D'une part, le maintien prolongé du two-shot au détriment du champ-contrechamp montre à merveille l'impossibilité des personnages de se débarrasser les uns des autres. D’autre part, le two-shot est combiné avec les paramètres du cadrage et de la mobilité de la caméra pour être utilisé de manière aussi appropriée que possible à l'agitation frénétique qui se dégage de nombreuses séquences. Parmi elles, la plus connue reste sans doute la scène du restaurant où David (Cary Grant) et Susan (Katherine Hepburn) se déchirent l'un après l'autre leurs vêtements dans le dos, ce qui oblige le premier à se coller à la seconde pour couvrir sa nudité partielle et les deux à quitter le restaurant comme deux pantins mécaniques. L'alternance de plans moyens et de plans américains, puis l'extrême mobilité de la caméra qui suit les personnages à travers leurs déplacements dans le restaurant, assurent une représentation visuelle du conflit infiniment plus efficace que n'aurait permis l'emploi du champ-contrechamp. Celui-ci, en effet, est beaucoup moins apte à montrer la position respective des personnages les uns par rapport aux autres et à faire sentir le regard que posent sur eux d'autres personnages présents dans le même espace.

\section{Le two-shot comme utopie du partage?}

Orlando de Sally Potter (1992), une adaptation du roman éponyme de Virginia Woolf, est l'histoire d'un personnage immortel dont la quête de liberté et d'amour nous fait traverser les grands moments de l'histoire anglaise et européenne, de la cour d'Elisabeth I Ie à la société contemporaine. Changeant de sexe au cours de son périple, Orlando découvre l'amour avec l'aventurier Shelmerdine, l'avant-dernière étape de son voyage à travers le temps. Bien que la plupart des «chapitres» de ce film posent des problèmes très intéressants du point de vue de la représentation du dialogue, ce passage est une belle illustration des manières très variées, mais en même temps très homogènes, du dépassement des clichés hollywoodiens sur l'objet et le sujet du regard (élève de Jarman et visiblement familière des thèses de Mulvey, Potter a une démarche très brechtienne des jeux du regard). Le dialogue avec Shelmerdine qui prépare la découverte de l'amour physique par Orlando se divise en deux parties. 
Dans la première moitié, Potter a condensé un très grand nombre de techniques qui tendent toutes sans exception à mettre en question la structure traditionnelle du regard et de la parole, et surtout des rapports convenus entre eux. Le fragment commence de manière presque agressivement classique, avec d'abord un plan d'ensemble, puis une série de champscontrechamps qui épousent la répartition des répliques. Mais rapidement cette illusion de transparence (au niveau du dispatching de la parole) et de hiérarchie (au niveau du sujet qui regarde et de l'objet regardé) est perturbée, non pas à l'aide de ruptures brutales, mais au moyen de toute une série de transformations subtiles qui brouillent les frontières et finalement les différences mêmes. S’agissant d'abord des données profilmiques, force est de constater que nombre de stéréotypes sexuels sont inversés (le visage de Shelmerdine, par exemple, est particulièrement féminin, tout comme sa façon de parler). Quant aux aspects filmiques, Potter accorde beaucoup d'attention à ce que la représentation des deux personnages soit la même pour l'homme que pour la femme (en durée, distance, angle, etc.). L'intervention la plus spectaculaire, toutefois, se situe à hauteur des interventions postfilmiques, où le film se dérobe ingénieusement à l'alternative brutale du two-shot (avec tout ce qu'il suppose de virtuellement polémique) et du champ-contrechamp (avec tout ce qu'il entraîne de violence symbolique dans la structuration de l'identification du spectateur au sujet et sa réduction objectale de l'autre). D'abord Potter casse la coïncidence de la parole et du plan: les two-shots sont parfois des plans muets, cependant que le dialogue se donne parfois à entendre sans que l'on voie les personnages; les champscontrechamps cessent d'être motivés ensuite par l'alternance des répliques, le personnage qui écoute devenant aussi important que celui qui parle. Ensuite, la réalisatrice remplace aussi les effets de montage (par exemple le saut du champ au contrechamp et inversement) par une caméra mobile qui se déplace lentement d'un personnage à l'autre, toujours légèrement en avance ou en retard sur l'enchaînement des paroles (lesquelles se font souvent littéralement écho). L'effet visé paraît moins d'empêcher l'identification du spectateur avec l'un des personnages 
que d'insinuer les possibilités d'une identification: loin d'amener le spectateur masculin à s'identifier sadiquement au "héros " et d'imposer au spectateur féminin une identification masochiste à la victime féminine (dirait Mulvey...), l'Orlando de Potter autorise des identifications multiples et positives, qui transposent au niveau de la réception ce que vit le personnage polymorphe de la fiction.

La première partie du dialogue est achevée par une mesure plus draconienne de la part de Potter, qui semble vouloir annoncer très fort la mutation stylistique qui se prépare: avant de s'abandonner entièrement à Shelmerdine, Orlando regarde droit dans la caméra, longuement, puis dit qu'elle va "s'évanouir ". Suit alors une transition par montage à la deuxième moitié du dialogue où les deux personnages se retrouvent au lit. Le même jeu de l'antistéréotype se poursuit ici à plusieurs niveaux. Dans le jeu amoureux, c'est la femme qui caresse l'homme couché sous elle, par exemple. Le dialogue de cette nouvelle partie reste d'ailleurs longtemps muet: les personnages communiquent par signes, gestes comme regards, et à l'échange de paroles se substitue une musique fluide, sans nulle solution de continuité, ce qui diminue encore davantage les distances entre les deux personnages. L'effet filmique le plus remarquable se situe pourtant à hauteur du champ-contrechamp, qui se voit désigné ainsi comme le centre du dispositif traditionnel auquel se prend Sally Potter. En effet, quand se met en place une courte série de champs-contrechamps sur les deux amants, la représentation des deux personnages est tout sauf symétrique: les quelques plans sur Orlando sont à peine subjectifs (la distance comme l'angle de vue choisis s'éloignent trop des règles classiques de raccord pour qu'on puisse penser que la caméra adopte vraiment le point de vue de l'homme); par contre, dans les quelques plans sur Shelmerdine, la subjectivation est poussée à ses extrêmes limites. D'une part les images sont ici très clairement subjectives (elles sont focalisées par la femme). D'autre part, ces mêmes images reprennent, mais sur un mode très différent, la rupture diégétique déjà accomplie par le regard direct jeté à la caméra par Orlando à la charnière des deux parties du dialogue: Shelmerdine, que l'on sait regarder Orlando, regarde 
aussi directement la caméra, détournant parfois les yeux, comme intimidé par l'insistance du regard de l'autre. Ce plan est capital, car il montre que la coïncidence du point de vue du personnage et du point de vue de la caméra fonctionnent d'une manière tout à fait nouvelle: au lieu de construire un dispositif où la caméra se dissimule derrière le regard du personnage afin de mieux impliquer le spectateur dans la fiction, la structure choisie par Potter montre que la caméra est révélée par le regard du personnage (et qui plus est par le regard d'un personnage féminin, qui s'approprie aussi la caméra), non pas pour immerger le spectateur dans le jeu de la fiction, mais afin de lui faire sentir et la présence de la caméra (tout regard direct qu'un personnage de fiction lance vers la caméra tend à révéler celle-ci) et la violence terrible exercée par elle (Shelmerdine intimidé détourne le regard, non seulement du regard d'Orlando dans la fiction, mais aussi et surtout de la caméra, donc, du spectateur).

Ici aussi, une petite comparaison avec un autre film peut permettre de bien mettre en valeur ce qui se passe dans Orlando. Dans The Color Purple (Steven Spielberg, 1985; d'après le livre du même titre d'Alice Walker), la protagoniste Miss Celie découvre elle aussi l'amour, non hétérosexuel (elle est brutalisée par son mari), mais lesbien, à travers sa rencontre avec la chanteuse de blues Shug Avery. La scène où les deux femmes se rapprochent l'une de l'autre n'est pas sans analogie avec le fragment d'Orlando que nous venons d'analyser. Les deux personnages parlent d'abord de l'amour, se font des confessions, et finalement s'embrassent. Pourtant, les antinomies entre Orlando et The Color Purple sont aussi colossales. Non seulement parce que Spielberg s'arrête là où Potter commence (chez Spielberg, la sexualité est censurée par métonymie d'abord et par métaphore ensuite: la caméra quitte la bouche des personnages pour cadrer leurs mains, puis glisse vers un accessoire du décor qui tient littéralement lieu de ce qui ne peut être montré à l'écran), mais aussi et surtout parce que la manière de filmer le dialogue est on ne peut plus différent. D'un côté, Spielberg obéit à l'enchâ̂nement mainstream du two-shot suivi d'une série de champscontrechamps au moment où l'action s'intensifie, pour terminer par un climax "poétique» dont les personnages sont absents (ils 
sont «relevés» par le décor symbolique). De l'autre, les champscontrechamps sont sans exception des plans filmés en trois quarts "par-dessus l'épaule" (over the shoulder shot), ce qui donne un petit côté voyeuriste à la scène et en même temps bloque toute possibilité de jeu sur la coïncidence virtuellement (et dans le cas d'Orlando, réellement) inquiétante de la coïncidence matérielle du regard du protagoniste et de celui de la caméra. Le voyeurisme du spectateur se trouve ainsi encouragé d'avance, puisqu'il peut rester en dehors des regards qu'échangent les deux femmes. Plus généralement, ce dispositif tend aussi à déplacer le centre de gravité de cette scène de l'aspect visuel des regards, relativement sous-exploité, au dialogue, lourdement mis en valeur. Spielberg, se dit-on, ne fait pas de film, il filme un scénario tiré d'un livre, et lui-même doit avoir senti le regard de l'auteure par-dessus son épaule.

Des quelques fragments examinés dans cet article, il est évidemment impossible de tirer des conclusions trop générales. Toutefois, l'analyse des dialogues filmés semble fonctionner à chaque fois comme une sorte de modèle réduit du film, ce qui ouvre sûrement des perspectives intéressantes à une nouvelle didactique du cinéma. Les exemples de Capra et de Hawks ont permis de montrer que le cinéma des années 1930 a su dépasser les contraintes "théâtrales» de la fixité de la caméra et d'un scénario très marqué par l'infratexte théâtral, sans pour autant casser les moules du cinéma narratif. Au contraire: les manières dont on voit Capra et Hawks "ruser» avec ces nouvelles contraintes sont tout à fait caractéristiques de l'établissement des nouvelles lois du montage dans le cinéma parlant. La comparaison des œuvres de Potter et de Spielberg de son côté a offert l'occasion de pointer, images en main si l'on ose dire, les différences entre deux manières de rendre le regard et la voix des femmes dans le cinéma commercial. Ces différences ont été soulignées jusqu'ici dans des analyses de type «idéologique». Il n'était pas inutile de les étayer aussi sur un plan plus formel, tout en les inscrivant dans une histoire plus longue du cinéma. En même temps, la confrontation des quatre films s'est montrée très révélatrice aussi quant à l'intérêt didactique de l'analyse des dialogues. Outre le fait que la sélection de ce genre de passages 
facilite la comparaison proprement dite (puisque tous les films narratifs, à quelques exceptions près, contiennent des dialogues, il ne faut jamais "forcer" la comparaison entre les œuvres qu'on cherche à rapprocher), le caractère tellement "évident» et «banal» d'un dialogue invite souvent le metteur en scène à y accorder un soin particulier. Le dialogue met alors en abyme une certaine esthétique (pour bien marquer ce point, qui aurait pu s'illustrer de manière crue avec, disons, Godard, on a choisi dans cet article des exemples relativement discrets, où les décisions et les a priori du metteur en scène ne s'avèrent pas moins visibles). Enfin, la lecture contrastée du two-shot et du champ-contrechamp a montré clairement qu'il n'est pas possible de maintenir l'interprétation traditionnelle de leur combinaison que proposent les théoriciens du montage invisible. Le two-shot est bien plus qu'une forme particulière de plan d'ensemble ou d'establishing shot. Le champ-contrechamp n'est pas seulement la technique à laquelle on passe une fois que s'enclenche l'action. Une lecture syntagmatique et paradigmatique de ces procédés filmiques est nécessaire, de manière à rendre compte de la complexité des films, qui sont toujours à la fois des illustrations de règles générales et d'inévitables cas d'espèce.

Université catholique de Leuven

\section{NOTES}

1. On peut penser ici à Robert McKee (1999), dont le livre est sans doute dans son genre le plus influent du moment. On se rappellera que Truffaut, dans sa polémique contre Aurenche et Bost, ne fait pas de distinction entre le scénario et le dialogue (Truffaut 2000).

2. On pense évidemment en tout premier lieu à Cavell (1981).

3. Citons, à titre d'exemple, le manuel classique de Bordwell et Thompson (1997).

4. Cf. l'entretien entre Éric Rohmer et Carole Desbarats : "Filmer la conversation" (Desbarats 1990, p. 134-135).

5. De très beaux exemples dans Chion 1982, toujours soucieux de l'irréductible singularité des œuvres qu'il scrute.

6. Voir plusieurs contributions dans Abel et Altman 2001.

7. Voir Bolter et Grusin 1999.

8. Voir Gaudreault et Marion 2000. La grande différence avec la théorie précitée de la "remédiation» est que le poids d'un type de représentation unique (le "réalisme») est ici heureusement absent et qu'on y trouve une articulation plus fine de la théorie des médias aux contextes socioculturels et institutionnels des formes discursives. 
9. Pour un exemple de ce genre d'analyse, voir la lecture du début du Faucon maltais dans Bordwell et Thompson 1997 (surtout p. 291).

10. Voir les études réunies dans Mulvey 1989.

11. Voir Cavell 1981.

12. Cette analyse s'oppose donc à celle de Bordwell et Thompson, qui choisissent dans leur Film Art un fragment du même film pour démontrer l'usage narratif, soit invisible ou naturalisé, du champ-contrechamp (Bordwell et Thompson 1997, p. 293).

\section{RÉFÉRENCES BIBLIOGRAPHIQUES}

Abel et Altman 2001: Richard Abel et Rick Altman (dir.), The Sounds of Early Cinema, Bloomington, Indiana University Press, 2001.

Bolter et Grusin 1999 : Jay David Bolter et Richard Grusin, Remediation. Understanding New Media, Cambridge, MIT Press, 1999.

Bordwell et Thompson 1997 : David Bordwell et Kristin Thompson, Film Art. An Introduction, New York, McGraw-Hill, 1997.

Cavell 1981 : Stanley Cavell, Pursuits of Happiness: The Hollywood Comedy of Remarriage, Cambridge, Harvard University Press, 1981.

Chion 1982: Michel Chion, La Voix au cinéma, Paris, Cahiers du Cinéma/Éditions de l'Étoile, 1982.

Desbarats 1990 : Carole Desbarats, Pauline à la plage d'Éric Rohmer, Liège, Yellow Now, 1990.

Gaudreault et Marion 2000: André Gaudreault et Philippe Marion, «Un média naît toujours deux fois... ", Sociétés \& Représentations, n 9, 2000, p. 21-36.

McKee 1999: Robert McKee, Story: Substance, Structure, Style and the Principles of Screenwriting, London, Methuen, 1999.

Mulvey 1989: Laura Mulvey, Visual and other Pleasures, Bloomington, Indiana University Press, 1989.

Truffaut 2000 : François Truffaut, «Une certaine tendance du cinéma français », dans Le Plaisir des yeux. Écrits sur le cinéma, Paris, Petite Bibliothèque des Cahiers du cinéma, 2000, p. 293-314.

Dis-moi comment tu filmes — et comment tu montes — tes dialogues et je te dirai quel genre de film tu réalises 